\title{
Low Prevalence of Vancomycin Resistant Enterococci Colonization in Intensive Care Patients in a Brazilian Teaching Hospital
}

\author{
Antonia T. Tresoldi, Luis Gustavo O. Cardoso, \\ Giane V.Castilho, Sônia R. P. E. Dantas, \\ Angela von Nowakonski, Ricardo M. Pereira \\ and Plínio Trabasso
}

\author{
Clinical Hospital, State University of Campinas; Medical Science \\ Faculty,State University of Campinas, SP, Brazil
}

\begin{abstract}
Vancomycin-resistant enterococci (VRE) are important pathogens involved in nosocomial infections. Colonization precedes infection and the number of colonized individuals is about 10 times higher than the number of infected patients. We examined VRE colonization in two intensive care units from October 2003 to June 2004. Perirectal swab specimens were obtained from all patients, starting on the $5^{\text {th }}$ day after admission, and then weekly. A total of 249 swabs were obtained from 112 patients. Nine patients had VRE-positive swabs, giving a positive rate of $8.0 \%$. The rate of patients colonized by V-R E. faecalis was $1.8 \%(n=2), 4.5 \%$ by V-R E. gallinarun $(n=5)$ and $1.8 \%$ by V-R E. casseliflavus $(n=2)$. No V-R E. faeciun was isolated. None of the patients that had been colonized by VRE were found to be infected by these pathogens. In summary, a low prevalence of colonization by VRE was found in our institution. Only a structured surveillance program, based on active searching, was able to detect this low number of cases.
\end{abstract}

Key Words: Vancomycin-resistant enterococci, VRE, colonization.

Vancomycin-resistant Enterococci (VRE) were isolated for the first time in the United Kingdom and in France, in 1986 [1,2]. Since then, an increasing number of patients have been reported to be colonized by these agents. The frequency of colonization varies widely, being 2 to $5 \%$ in France [3], 12\% in the Netherlands [4] and 20\% in Korea [5], mostly involving patients in intensive care units (ICU). In 1998, in a prospective study carried out in seven Norwegian hospitals, no E. faecalis or E. faecium resistant to vancomycin were detected [6]. VRE was first described in Brazil in 1996; however, variation in the prevalence of colonization in Brazilian hospitals is high, with reported rates from zero to $33 \%$ [7-9].

Beyond colonization, vancomycin-resistant E. faecalis and E. faecium are also important pathogens causing nosocomial infections, usually involving the urinary tract, surgical wounds or sepsis $[10,11]$. The most common risk factors for colonization/infection by VRE are ICU stay, prolonged use of antibiotics, especially vancomycin, third-generation cephalosporins and metronidazol, length of hospital stay, neutropenia and contact with another patient colonized or infected by VRE [9,12-17].

The presence of VRE was no longer being documented in our institution; so we decided to search for VRE colonization in patients in the intensive care units.

Received on 27 February 2006; revised 30 June 2006.

Address for correspondence: Dr. Antonia Teresinha Tresoldi. Rua José Paulino, 1875, apto 43-A, Campinas, SP, Zip code: 13013-002. E-mail:tresoldi@hc.unicamp.br.

The Brazilian Journal of Infectious Diseases 2006;10(4):239-241. (C) 2006 by The Brazilian Journal of Infectious Diseases and Contexto Publishing. All rights reserved.

\section{Material and Methods}

The Clinical Hospital of the State University of Campinas is a 400-bed, tertiary care, teaching hospital. The medicalsurgical intensive care unit (MSICU) for adults comprises 20 beds, and there are 10 beds in the pediatric intensive care unit (PICU). The study was carried out from October 2003 to June 2004 in the PICU and from January to October 2004 in the MSICU. Perirectal swab samples were taken from all patients, starting on the $5^{\text {th }}$ day after admission and then on a weekly basis until discharge or death.

Samples were inoculated into Esculin Bile Agar with vancomycin $(6 \mu \mathrm{g} / \mathrm{mL})$ and aerobically incubated for $48 \mathrm{~h}$ at $35^{\circ} \mathrm{C}$; growing colonies were then transferred to a Blood Agar plate and re-incubated for $24 \mathrm{~h}$. After that, the identification of the microorganism at the species level and the antibiotic susceptibilities were determined using the Vitek system (BioMérieux-Vitek). The colonies not identified by the Vitek system were submitted to motility and pigmentation tests. Vancomycin resistance was confirmed using Kirb-Bauer disk diffusion and the E-test.

Demographics (sex, age, length of stay in the ICU), risk factors (mechanical ventilation, central venous catheter, indwelling urinary catheter, antibiotic used) and outcome (discharge or death) data were collected. The data were analyzed using SPSS software (Version 10.0; SPSS Inc., Chicago.).

The institutional Ethics Committee approved the study and written consent was obtained from patients or relatives before enrollment.

\section{Results}

Two-hundred-forty-nine samples from 112 patients were obtained, with a mean of 2.2 samples/patient (range: 1 to 12 
samples/patient). Demographics and risk factors data are presented in Table 1.

Table 1. Demographics and risk factors data. PICU - pediatric intensive care unit; MSICU - medical-surgical intensive care unit for adults

\begin{tabular}{lcc}
\hline Characteristic & PICU & MSICU \\
\hline Number of patients & 47 & 65 \\
Median samples/patients & 3.0 & 1.6 \\
Median age & 39 months & 47 years \\
Median length of stay & 30 days & 19 days \\
Central Venous Catheter & $38(90.9 \%)$ & $60(93.2 \%)$ \\
Mechanical ventilation & $43(91.5 \%)$ & $65(100.0 \%)$ \\
Indwelling urinary catheter & $20(42.6 \%)$ & $65(100.0 \%)$ \\
Surgery & $12(25.5 \%)$ & $34(52.2 \%)$ \\
\hline
\end{tabular}

During the hospital stay, 38 (90\%) patients of the PICU used antibiotics, cefazolin, amikacin and ampicillin being the most frequently-used drugs, in decreasing order. In the MSICU, 65 (98\%) patients used antibiotics, the most frequently used being cefepime, vancomycin and imipenem.

The results of cultures for VRE are presented in Table 2.

Table 2. Results of the cultures. PICU - pediatric intensive care unit; MSICU - medical-surgical intensive care unit of adults

\begin{tabular}{|c|c|c|c|}
\hline Cultures & PICU(n) & MSICU (n) & Total (n) \\
\hline Negatives & 43 & 60 & 103 \\
\hline $\begin{array}{l}\text { V-R E. faecalis } \\
(\mathrm{MIC}>32 \mu \mathrm{g} / \mathrm{mL})\end{array}$ & 1 & 1 & 2 \\
\hline V-R E. gallinarum* & 2 & 3 & 5 \\
\hline $\begin{array}{l}\text { V-R E. casseliflavus } \\
\text { (MIC } 8 \mu \mathrm{g} / \mathrm{mL})\end{array}$ & 1 & 1 & 2 \\
\hline Total & 47 & 65 & 112 \\
\hline
\end{tabular}

V-R E.: Vancomycin resistant Enterococcus.

*3 strains with minimum inhibitory concentration (MIC) $>32 \mu \mathrm{g} / \mathrm{mL}, 1$ strain with $\mathrm{MIC}=16 \mu \mathrm{g} / \mathrm{mL}$ and 1 strain with $\mathrm{MIC}=8 \mu \mathrm{g} / \mathrm{mL}$.

No V-R E.faecium was isolated in our study. The rate of patients colonized by VRE was $8.0 \%$; the rate of patients colonized by V-R E. faecalis was $1.8 \%$; V-R E. gallinarun accounted for $4.5 \%$ and V-R E. casseliflavus $1.8 \%$. There was no discordance among the results of different samples from the same patient; in patients with two or more positive cultures, the same microorganism was found in all of them.

Only two patients were found to be colonized by V-R E. faecalis. The firstwas an infant with fulminant hepatitis, who had already stayed for a month in another hospital and was transferred to our institution for a liver transplantation; unfortunately he died on the $5^{\text {th }}$ day after admission. The second patient was a woman with acute intermittent porphyria, who stayed in the MSICU for more than three months; during her hospitalization, she used various antibiotics (these being cefepime, vancomycin, amphotericin B, and piperacillin + tazobactam). VRE was identified during the last six weeks of her stay in the MSICU, and the patient was treated in a way to avoid contact contamination, ever since the first documented positive culture. Neither of these colonized patients were found to be infected by VRE. The V-R E. faecalis of these two patients was susceptible to ampicillin and resistant to teicoplanin. Screening of contacts of these patients colonized by V-R E. faecalis did not demonstrate cross transmission or secondary cases.

\section{Discussion}

We found a low incidence of V-R E. faecium and E. faecalis in the Clinical Hospital of the State University of Campinas. Furthermore, it is possible that one of these patients was already contaminate with VRE when he was transferred from another hospital. In the second case, with a long period of stay after colonization, we consider that the contact precautions were effective in order to prevent dissemination in the MSICU. The absence of cross-contamination could also have contributed to the low incidence of colonization, especially in the MSICU. Studies have shown that crosscontamination is the main mechanism of transmission of VRE in hospitals [18,19], and that, since this pathogen is introduced in the environment, the tendency is that it becomes endemic if effective measures for control are not taken [20]. Active surveillance has an important role in order to identify both patients-at-risk and carriers of VRE, and it is necessary to take precautions to avoid contamination by contact. Various studies have shown that the incidence of colonized individuals is about 10 times higher than the frequency of infected patients, who would be more dangerous for cross-contamination [21]. Although an analysis of risk factors for colonization could not be performed because of the small number of cases, we note that even though there were differences in the use of antibiotics in the two ICUs, especially vancomycin, there were similar frequencies of colonization. Our study population presented almost all of the risk factors related to colonization by VRE described in other studies. Such low incidences of colonization had already been described in other tertiary hospitals, including hospitals in Brazil [8].

We identified seven cases of VRE involving species that are considered to have low pathogenicity for humans, including E. casseliflavus $(\mathrm{n}=2)$ and E. gallinarum $(\mathrm{n}=5)$. The high prevalence of these resistant species, in comparison to $E$. faecalis and E. faecium, had already been described by other authors [8,22,23]. Recently E. gallinarum and E. casseliflavus have been found to be associated with invasive infections, including bacteremia and infection of the central nervous system [24-27]. However, these types of infections, especially the bacteremias, are associated with a low risk of mortality [28].

In summary, we found a low prevalence of colonization by VRE in our hospital. Only a structured surveillance program, based on active search, was able to detect the very few cases; this type of investigation should be carried out routinely in all units where there are patients -at risk. Infection-control 
practitioners must be on alert for the occurrence of nosocomial infections caused by E. gallinarum or E. casseliflavus resistant to vancomycin, since these bacteria have been identified in hospital patients.

\section{Acknowledgements}

G.V.C. was supported by student grants from FAPESP (03/ 06372-5)

\section{References}

1. Uttley A.H., Collins C.H., Naidoo J., et al. Vancomycin-resistant enterococci. Lancet 1988;1:57-8.

2. Leclercq R., Derlot E., Duval J., et al. Plasmid-mediated resistance to vancomycin and teicoplanin in Enterococcus faecium. N Engl J Med 1988;319:157-61.

3. Boisivon A., Thibault M., Leclerq R. Colonization by vancomycin-resistant enterococci of the intestinal tract of patients in intensive care units from French general hospitals. Clin Microbiol Infect 1997; 3:175-9.

4. Van den Bogard A.E., Mertens P., London N.H., et al. High prevalence of colonization with vancomycin and pristinamycin-resistant enterococci in healthy humans and pigs in The Netherlands: is the addition of antibiotics to animal feed to blame? J Antimicrob Chemother 1997; 40:4546.

5. Seong C.N., Shim E.S., Kim S.M., et al. Prevalence and characterization of vancomycin-resistant enterococci in chickens intestines and humans of Korea. Arch Pharm Res 2004; $27: 246-53$.

6. Simonsen G.S., Andersen B.M., Digranes A., et al.. Low Faecal Carrier Rate of Vancomycin Resistant Enterococci in Norwegian Hospital Patients. Scand J Infect Dis 1998;30:465-8.

7. Dalla Costa L.M., Souza D.C., Martins L.T., et al. Vancomycinresistant Enterococcus faecium: First case in Brazil. Braz J Infect Dis 1998;2:160-3.

8. Maschieto A., Martinez R., Palazzo I.C.V., et al. Antimicrobial resistance of Enterococcus sp. Isolated from the Intestinal Tract of Patients from a University Hospital in Brazil. Mem Inst Oswaldo Cruz 2004;99:763-67.

9. Furtado G.H.C., Martins S.T., Coutinho A.M., et al. Prevalence and Factors Associated with Rectal Vancomycin-resistant enterococci Colonization in Two Intensive Care Units in São Paulo, Brazil. Braz J Infect Dis 2005;9:64-69.

10. Edmond M.B., Ober J.F., Dawson J.D., et al. Vancomycinresistant enterococcal bacteremia: natural history and attributable mortality. Clin Infect Dis 1996; 23:1234-9.

11. Furtado G.H.C., Martins S.T., Coutinho A.P., et al. Incidência de Enterococcus resistente à vancomicina em hospital universitário no Brasil. Rev Saúde Pública 2005;39:41-6.

12. Broadhead J.M., Parra D.S., Skelton P.A. Emerging multiresistant organisms in the ICU: epidemiology, risk factors, surveillance and prevention. Crit Care Nurs Q 2001;24:20-9.

13. Husni R., Hachem R., Hanna H., et al. Risk factors for vancomycin-resistant Enterococcus (VRE) infection in colonized patients with cancer. Infect Control Hosp Epidemiol 2002;23:102-3.
14. Suntharam N., Lankford M.G., Trick W.E., et al. Risk factors for acquisition of vancomycin-resistant enterococci among hematology-oncology patients. Diagn Microbiol Infect Dis 2002; 43:183-8.

15. Carmeli Y., Eliopoulos G.M., Samore M.H. Antecedent treatment with different antibiotic agents as a risk factor for vancomycin-resistant Enterococcus. Emerg Infect Dis 2002;8:802-7.

16. Zaas A.K., Song X., Tucker P., et al. Risk Factors for development of vancomycin-resistant enterococcal bloodstream infection in patients with cancer who are colonized with vancomycinresistant enterococci. Clin Infect Dis 2002;15:1139-46.

17. Ho P.L. Carriage of methicillin-resistant Staphylococcus aureus, ceftazidime-resistant Gram-negative bacilli, and vancomycinresistant enterococci before and after intensive care unit admission. Crit Care Med 2003;31:1175-82.

18. Bonten M.J.M., Hayden M.K., Nathan C., et al. The epidemiology of patient colonization and environmental contamination with vancomycin-resistant Enterococcus: the challenge of infection control. Lancet 1996;348:1615-9.

19. Воусе J.M. Vancomycin-resistant enterococcus. Detection, epidemiology and control measures. Infect Dis Clin North Am 1997; 11:367-84.

20. Morris J.G., Shay D.K., Hebden J.N., et al. Enterococci resistant to multiple antimicrobial agents, including vancomycin: establishment of endemicity in a university medical center. Ann Intern Med 1995;123:250-9.

21. Montecalvo M.A., De Lencastre H., Carraher M., et al. Natural history of colonization with vancomycin-resistant Enterococcus faecalis. Infect Control Hosp Epidemiol 1995; $16: 680-5$

22. Christidou A., Nikolaids P., Skoutelis A., et al. Vancomycinresistant enterococci in Greece: a multicentre prevalence study on intestinal colonization. Cli Microb Infection 2005;11(suppl 2):223.

23. Barbosa D., Lima L., Silbert S., et al. Evaluation of the prevalence and isk factors for colonization by vancomycin-resistant Enterococcus among patients on dialysis. Am J Kidney Dis 2004;44:337-43.

24. Ratanasuwan W., Iwen P.C., Hinrichs S.H., et al. Bacteremia due to motile Enterococcus species: clinical features and outcomes. Clin Infect Dis 1999;28:1175-7.

25. Kurup A., Tee W.S.N., Loo L.H., et al. Infection of central nervous system by motile Enterococcus: first case report. J Clin Microbiol 2001;39:820-2.

26. Reid K.C, Cockerill I.F., Patel R. Clinical and epidemiological features of Enterococcus casseliflavus/flavescens and Enterococcus gallinarum bacteremia: a report of 20 cases. Clin Infect Dis 2001;32:1540-6.

27. Yoko T., Keisuke S., Tohru A. Meningitis caused by Enterococcus gallinarum in patients with ventriculoperitoneal shunts. J Infect Chemoter 2003;9:348-50.

28. Choi S.H., Lee S.O., Kim T.H., et al. Clinical Features and Outcomes of Bacteremia caused by Enterococcus casseliflavus and Enterococcus gallinarum: Analysis of 56 cases. Clin Infect Dis 2004; 38:53-61. 